\title{
Practical Electroanalytical Measurements in Liquid Cell TEM
}

\author{
Khim Karki ${ }^{1}$, Daan Hein Alsem ${ }^{1}$, and Norman Salmon ${ }^{1}$
}

${ }^{1}$ Hummingbird Scientific, Lacey, WA, USA.

Recent progress in liquid cell transmission electron microscopy (LC-TEM) has enabled dynamic observation of materials behavior in their native reaction environment with higher spatial-and-temporal resolution [1] However, the inability to acquire true quantitative information, reminiscent of bulk behavior, has limited its use in the broader applications such as electrochemistry, photocatalysis, and other energy related devices [2,3]. Particularly, the liquid electroanalytical measurements performed during the studies such as battery operation, metal nucleation and growth during electrodeposition, dendrite formation, and metal corrosion, etc., can benefit from the reliability and the repeatability of data - comparable to bulk value. The development of liquid cell microscopy platform capable of achieving true electroanalytical measurements is highly desirable.

The challenges associated with replicating bulk-scale electrochemistry data in the environmental cell microscopy platform are well-known [1]. First, the hardware components are not best optimized to perform in the reduced scale environment of the TEM [4]. Second, the chips configurations such as sizes and aspect ratios of different electrodes suitable for various electroanalytical measurements are poorly designed. Here, we present an operando liquid cell TEM microscopy platform that enables accurate and reliable electroanalytical measurements using some model compounds, mimicking bulk behavior.

The schematic and the platform for liquid cell microscopy are presented in Figure 1A and Figure 1B, respectively. The liquid cell platform typically consists of two microfabricated chips sandwiched with electron transparent $\mathrm{SiNx}$ membranes for encapsulating liquid and viewing in the microscope. The electrochemical cell with an advanced hardware system and optimized electrochemistry chips with a specialized configuration of working electrode (WE), counter electrode (CE) and reference electrode (RE) is integrated in the hardware around the cell. This new configuration allows true quantitative measurements of electrochemical processes with details resembling the complete cycle of the bulk. We have developed an advanced liquid cell TEM platform which can allow wide range of electrochemical measurements such as batteries, metal electrodeposition, corrosion, and photocatalysis, etc. As an illustration, we present cyclic voltammetry studies of $01 . \mathrm{M} \mathrm{CuSO}_{4}$ and $20 \mathrm{mM} \mathrm{K} 3 \mathrm{Fe}(\mathrm{CN})_{6} / 20 \mathrm{mM}$ $\mathrm{K}_{4} \mathrm{Fe}(\mathrm{CN})_{6}$ in $0.1 \mathrm{M} \mathrm{KCl}$ solutions as case studies. In the case of $0.1 \mathrm{M} \mathrm{CuSO}_{4}$, the copper deposition and stripping occurs at the working electrode at distinct redox peaks in liquid cell (Figure 1C) and the result mimics the bulk electrochemical cells (Figure 1D) with large electrode areas and larger volume of electrolyte solution [5]. This work highlights the fact that with suitable hardware integration and with better layout of the patterned chips configuration, bulk behavior of the electrochemical processes can be both observed and measured quantitatively. These results demonstrated for small volume and limited diffusion cell geometries mimicking the bulk data can be further implemented in the quantitative measurements of broader electrochemical systems including photocatalysis using the next generation optical liquid operando microscopy systems, as will be discussed [6].

\section{References:}

[1] F.M. Ross in "Liquid Cell Electron Microscopy", Cambridge University Press (2016). 
[2] J. Lim et al, Science 353 (2016), p. 566.

[3] N. Hodnik et al, Acc. Chem. Res. 49 (2016), p. 2015.

[4] E. Fahrenkrug et al, Journal of Electrochemical Society 164 (2017), p. H358.

[5] D. Grujicic and B. Pesic, Electrochimica Acta 47 (2002), p. 2901.

[6] KK, DHA, and NS acknowledge funding from the Department of Energy, Office of Basic Energy Sciences, SBIR Grant \# DE-SC0009573.

A

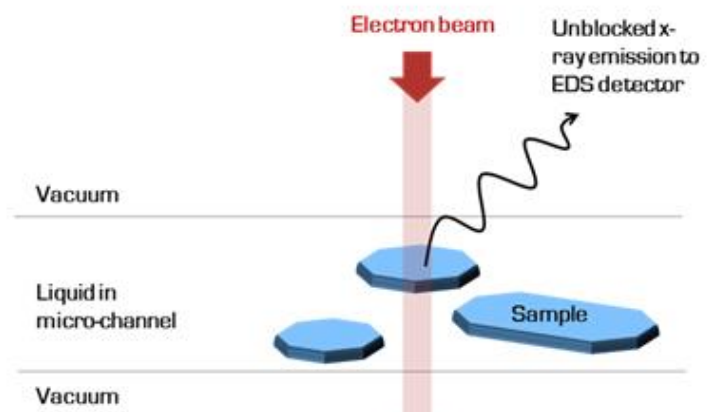

C

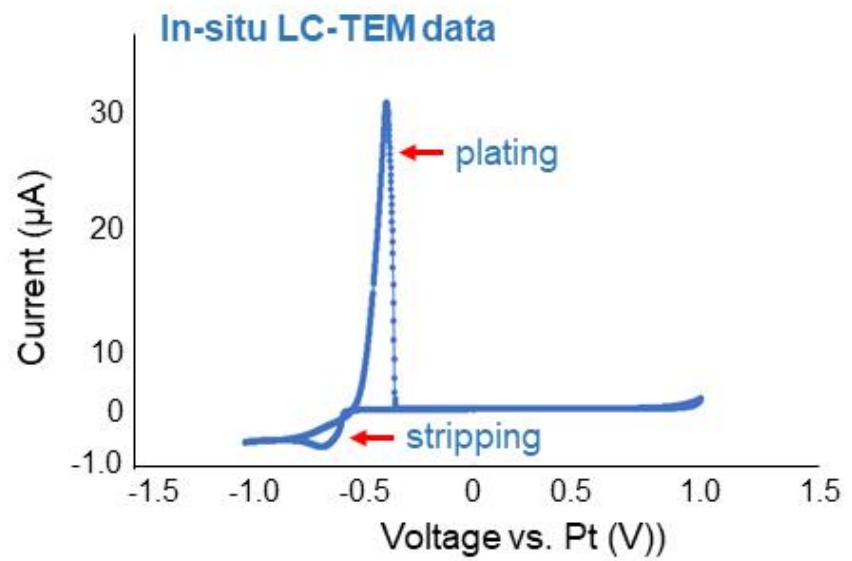

B

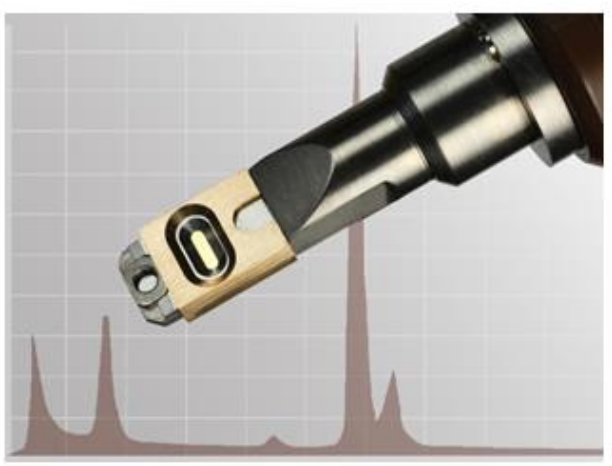

D

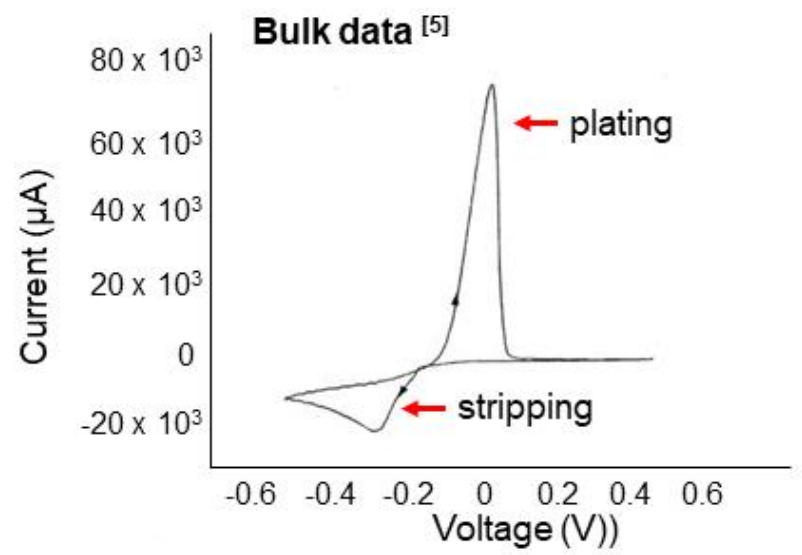

Figure 1. (A) Schematic of assembled liquid cell. (B) Liquid cell tip with EDS compatibility. (C) Cyclic voltammogram of $0.1 \mathrm{M} \mathrm{CuSO}_{4}$ performed in (C) in-situ LC-TEM, and (D) bulk container extracted from Ref 5. 